\title{
Plasmonic nanosurgery for destruction of microscopic residual disease following resection in
}

\section{cancer}

\begin{abstract}
Surgery, when possible, continues to remain the best possible treatment option for cancer patients. However, in some cases the cancer can be unresectable and requires radiation or chemotherapy prior to surgery even being an option. Macroscopic surgery can leave behind microscopic residual disease, which can lead to recurrence and may be lethal. Image-guided surgery has shown clinical benefit by reducing recurrence rates and improving survival in patients. Remotely triggered destruction of microscopic residual disease immediately following macroscopic resection would be an attractive tool for surgeons and a beneficial option for patients. Externally activated, targeted destruction of cancer could also be used for the management of unresectable tumors. Here, we explore the use of biocompatible, gold nanoparticles for photo-triggered destruction of microscopic residual disease through the formation of plasmonic nanobubbles. A pulsed laser is used to activate gold nanoparticles, which through surface plasmon resonance causes a rise in temperature that in turn vaporizes nearby liquid medium to form short-lived nanobubbles, which are referred to as plasmonic nanobubbles. When these nanobubbles form and then collapse, a pressure pulse is emitted only in cancerous cells that can be photo acoustically detected and this signal can be converted into an image. These nanobubbles can be used either for nanoguided surgery or nanosurgery in which the pressure pulse emitted actually destroys the cancer cells. These plasmonic nanobubbles have been extensively explored by Lukianova-Hleb and co-workers for cancer applications. Their animal studies showed that following surgery, they could detect the remaining tumor cells at a tissue depth of $4 \mathrm{~mm}$, which is highly significant for the clinical translation of this technology. The high selectivity of plasmonic nanobubbles, along with the ability for real-time elimination of microscopic residual disease demonstrated by these researchers could form the basis for a clinical trial and the eventual clinical translation of nanosurgery for cancer applications.
\end{abstract}

Keywords: nanotechnology, diagnostics, theranostics, oncosurgery, photo-triggered, laser ablation, imaging-guided surgery, real-time detection, surface plasmon resonance, oncology, cancerous, angiography, NIR imaging, nanoclusters, picoseconds
Volume 4 Issue I - 2017

\author{
Derek VanDyke, Shailee Paliwal, Prakash Rai \\ Department of Chemical Engineering, University of \\ Massachusetts Lowell, USA
}

Correspondence: Prakash Rai, Department of Chemical Engineering, University of Massachusetts Lowell, University Ave, Perry Hall 2I2, Lowell, MA, United States, Tel 978-934-497I, Email prakash_rai@uml.edu

Received: September 27, 2016 | Published: February 28,

2017
Abbreviations: EGFR, epidermal growth factor receptor; HNSCC, head and neck squamous cell carcinoma; ICG, indocyanine green; NIR, near-infrared; MRD, microscopic residual disease, PNB, plasmonic nanobubbles, QD, quantum dot; SERS, surface enhanced raman scattering; SPR, surface plasmon resonance

\section{Introduction}

Cancer is one of the leading causes of death in the United States and its variable characteristics make it difficult to diagnose and treat Traditional methods of cancer therapy include, but are not limited to, radiation therapy, immunotherapy, and surgery. Currently, preoperative near-infrared (NIR) fluorescence or X-ray imaging is commonly used to highlight tumor location inside the body. However, during surgery, a surgeon is limited to his or her own visual and tactile acuity to discriminate between cancerous and healthy tissue, which leads to resection of healthy tissue that may be essential for organ function. As a result, intra operative NIR imaging was developed, in which fluorescing agents, such as ICG, are released into the bloodstream to accumulate in and around the tumors and can then be imaged during surgery to differentiate cancer cells. ${ }^{1}$ Despite such advancements in surgical technology, complete removal of cancerous cells with minimal damage to healthy tissue is still limited by the surgeon's precision as well as the fact that residual cancer cells, referred to as microscopic residual disease (MRD), are nearly always left behind. In a disease such as cancer, even a single tumor-initiatingcell left behind has the potential to regrow and metastasize, which could lead to lethal recurrence, essentially negating any previous treatment methods. ${ }^{2}$

Nanotechnology provides a means to destroy MRD with minimal damage to surrounding tissue. Nanoparticles with both therapeutic and diagnostic applications can be a valuable aid in surgical oncology. Semiconductor quantum dots (QD), surface-enhanced Raman scattering (SERS) colloidal gold nanoparticles, as well as tumor targeting ligands are undergoing extensive research and show preclinical potential for use in intraoperative imaging as well as for cell destruction by triggered therapy, such as light, sound, or radiation triggered. ${ }^{3}$ This allows for both nano-guided surgeries 
in which nanoparticles are used to detect cancerous cells, and for nanosurgery in which the nanoparticles are used to help remove MRD. Additionally, nanotechnology allows for selective cancer cell targeting eliminating harm to normal tissue. ${ }^{4}$ By providing a means of intraoperatively imaging, these nanoprobes increase surgical resection efficiency, and treat MRD when followed by a remotely triggered therapy. In this mini-review, we investigate the use of biocompatible, gold nanoparticles for laser-triggered destruction of MRD through the formation of plasmonic nanobubbles (PNB).
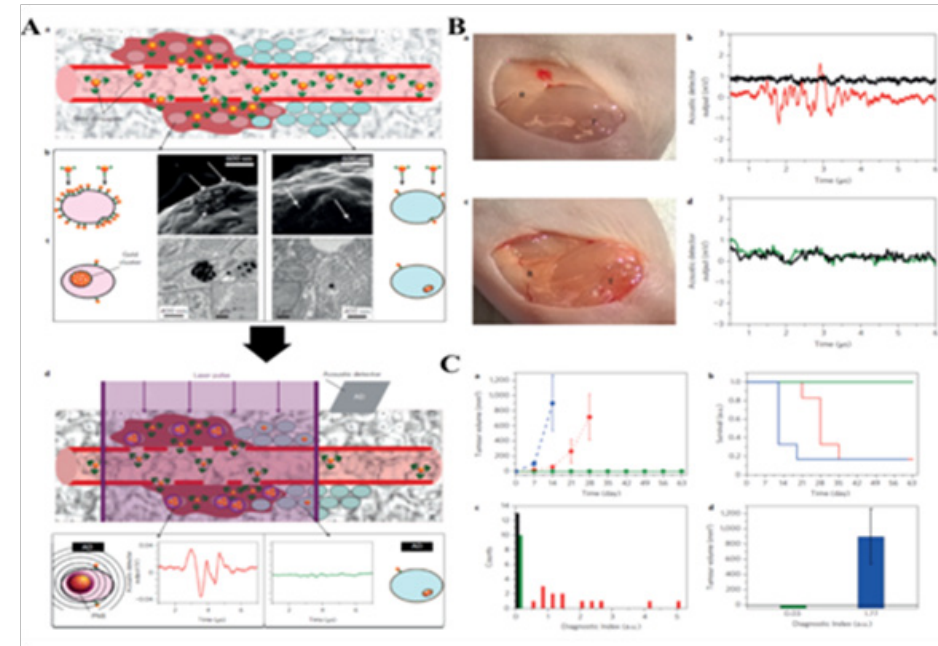

Figure IA: (a) Systemic delivery of gold conjugates to the tumor via their leaky vasculature (b) Accumulation of gold conjugates by receptors of cancer cells (gold shown with white arrows in illustrative scanning electron microscopy images) (c) Intracellular clustering of gold conjugates via receptor-mediated endocytosis (illustrative transmission electron microscopy images). A gold cluster, on exposure to a single laser pulse of low fluence, selectively generates a PNB only in cancer cells; normal cells with non-specifically internalized single gold nanoparticles do not generate PNBs because of the higher threshold of PNB generation (d) The acoustic signal of a PNB (illustrative red time response) reports even a single cancer cell in solid tissue, but not normal cells (illustrative green time response).

Figure IB PNBs intraoperatively detect MRD in a surgical bed and guide its resection in real time with standard surgery. (a) Image of the surgical bed after primary surgery. T, MRD-positive location in the nest of the primary tumor; R, MRD-negative location with normal tissue. (b) Acoustic time responses to single laser pulses $(782 \mathrm{~nm}, 30 \mathrm{ps}, 70 \mathrm{~mJ} \mathrm{~cm}$-2 obtained immediately after primary surgery at the 'T' location of possible MRD (red) and at the MRD negative location ' $R$ ' (black). (c) Image of the surgical bed after PNB-guided surgery. (d) Acoustic time responses obtained after PNB-guided surgery in the location of the secondary resections (green) and in the initially MRD-negative location (black).

Figure IC PNBs improve surgical outcome in both resectable and unresectable MRDs. The animal group-averaged metrics of local recurrent tumors after standard surgery with resectable MRD (blue, $n=6$ ), PNB-guided surgery of resectable MRD (green, $n=5$ ) and PNB nanosurgery of unresectable MRD (red, $n=6$ ) show a significant improvement in the outcome in both resectable and unresectable cases when the surgery is enhanced with PNBs. (a) Tumor volume. (b) Animal survival. (c) Histograms of the Diagnostic Index obtained in MRD-positive (red) and -negative (black) locations after standard surgery and for MRDpositive locations after PNB-guided surgery (green). (d) Recurrent tumor volumes plotted for the group-averaged Diagnostic Indices after standard (blue) and PNB-guided (green) surgery show the prognostic potential of PNBs to intraoperatively predict tumor recurrence. Data are mean \pm standard error. Error bars are based on six (blue and red) or five (green) mice per group. Figure and legend reproduced with permission from Nature Publishing Group. ${ }^{2}$

\section{Discussion}

Metallic nanoparticles like those of gold or silver are frequently used as nanosized probes that can absorb and convert photons with sub wavelength resolution into heat through the process of surface plasmon resonance (SPR). One example of this was demonstrated by Lukianova-Hleb et al. in which they used targeted gold nanoparticles to form PNBS. These PNBs were then used for both PNB-guided surgery and PNB-based nanosurgery to more effectively destroy resectable and unresectable MRD respectively. These PNBs, a photomechanical product of light irradiation, show promise in both the intraoperatively detection and destruction of residual disease in vivo in head and neck squamous cell carcinoma (HNSCC) in real time. To synthesize these PNBs, first, $60 \mathrm{~nm}$ gold nanoparticles, conjugated to the cancer-specific antibody Panitumumab, are injected into the tumor site. The clinically approved antibody, Panitumumab is specific to the epidermal growth factor receptor (EGFR), which is over expressed on several solid cancers. The antibody conjugated gold nanoparticles are delivered to the tumor site due to the leaky vasculature of the tumor and the enhanced permeability and retention effect. In cancerous cells, due to receptor-mediated endocytosis, the gold nanoparticles begin to form clusters of about $300 \mathrm{~nm}$. The next step is to subject these gold clusters to a single laser pulse of low light dose. The gold nano clusters absorb a short NIR pulse and convert its photon energy to heat that evaporates nearby liquid medium into inflating and deflating nanobubbles, with the lifespan of about a nanosecond. Because the nano clusters are subjected to light irradiation for only a short period of time ( 30 picoseconds), there is not enough time for the cluster to absorb the light and heat up the gold nanoparticles as seen in photo thermal therapy. For normal cells, only single particles or small clusters of particles are formed inside the cells. Therefore, when subjected to the laser pulse there is not enough energy to form nanobubbles because for single particles or small clusters the PNB generation threshold is much higher. This allows for PNBs to have specificity only towards cancerous cells. During the formation/ collapsing of the nanobubbles, there is a pressure pulse that is emitted and acoustically detected allowing for nano-guided surgery. ${ }^{2}$ 
These nanoparticles were used for two different cases in vivo in mice with HNSCC: standard surgery plus PNB-nanosurgery in unresectable MRD, and for PNB-directed surgery in resectable disease. For both cases, once the primary tumor was removed, the surgical bed was probed with PNBs and the resulting acoustic signals were recorded in real-time. For unresectable disease, no further resections could be done to remove MRD after PNB-detection; therefore, the only destruction of MRD was through the mechanical impact of PNBs (i.e. PNB-nanosurgery). If the gold nano lusters are large enough, the mechanical impact of the PNBs being formed and collapsing can actually explode the cancer cells. In the nanoclusters do not reach this minimal size in non-cancerous cells, there is no cell destruction, demonstrating the high selectivity of PNB-nanosurgery. This study showed that PNB nanosurgery not only delayed tumor recurrence but improved animal survival by twofold compared to normal surgery. For the case of resectable MRD, after primary tumor resection, the surgical bed was scanned using PNBs to find any MRD positive areas. If any MRD positive locations were found, that location was further resected $1 \mathrm{~mm}$ and rescanned using PNBs (PNB-guided surgery). This procedure was continued until the area became MRD negative. For the case of PNB-guided surgery, this study showed that there was no recurrence observed and there was a tumor free survival for $100 \%$ of the treated mice. These PNB studies show that both resectable and unresectable MRD can be detected in vivo with high speed and cancer cell selectivity, and both PNB-nanosurgery and PNB-guided surgery lead to a better outcome compared to normal surgery techniques. ${ }^{2}$ This study shows that there is promise for these PNBs to be used for clinical PNB-guided surgery and PNB-nanosurgery.

\section{Conclusion}

The PNBs described above illustrate the ability for nanotechnology to increase the efficacy in removal of MRD either through nanoguided surgery or through nanosurgery. The above method illustrated a cancer cell sensitive nanotechnology that effectively generated PNBs that could be used for effective PNB-guided surgery and PNB-nanosurgery. Additionally, the PNBs could detect residual tumor cells deep in tissue at thicknesses of $4 \mathrm{~mm}$, which would be of significance in the clinical translation of these PNBs. The high selectivity of nanotechnology as seen in the case of PNBs, along with the ability for real-time elimination of MRD aids in the clinical translation of nanosurgery. In the clinic, these PNBs or other nanoguided surgeries could be incorporated with a surgical endoscope or a robotic arm therefore allowing for highly effective and quick methods for the detection and destruction of MRD. ${ }^{2}$ Some questions about the toxicities of these transient PNBs to normal cells remain. Also the efficiency of these nanoplasmonic procedures for destruction of cancer cells need to be improved. Despite several challenges that lie ahead for the clinical translation of these nanosurgery techniques, these PNBs show promise as an effective alternative method of detecting and removing MRD in cancer.

\section{Acknowledgements}

The authors acknowledge funding from the National Cancer Institute, a part of the National Institutes of Health (Award \# R00CA153948).

\section{Conflict of interest}

The author declares no conflict of interest.

\section{References}

1. Vahrmeijer AL, Hutteman M, Van der Vorst JR, et al. Image-guided cancer surgery using near-infrared fluorescence. Nature Reviews. Clinical Oncology. 2013;10(9):507-518.

2. Lukianova-Hleb EY, Shin Kimt Y, Belatsarkouski I, et al. Intraoperative diagnostics and elimination of residual microtumors with plasmonic nanobubbles. Nature Nanotechnology. 2016;11:525-532.

3. Singhal S, Nie S, Wang MD. Nanotechnology applications in surgical oncology. Annual Review of Medicine. 2010;61:359-373.

4. Davis ME, Chen Z, Shin DM. Nanoparticle therapeutics: an emerging treatment modality for cancer. Nature Reviews. 2008;7:771-782. 